\title{
qSOFA score and LqSOFA score as predictors of outcome in an elderly population with chest infection treated in the Emergency Department. A case series
}

\author{
Dimitrios Velissaris, ${ }^{1}$ Vasileios Karamouzos, ${ }^{2}$ Nikolaos Dimitrios Pantzaris, ${ }^{3}$ Loukas Kakoullis, ${ }^{4}$ \\ Charalampos Pierrakos, ${ }^{5}$ Menelaos Karanikolas, ${ }^{6}$ Charalampos Gogos ${ }^{1}$ \\ ${ }^{1}$ Department of Internal Medicine, University Hospital of Patras, Patras, Greece; ${ }^{2}$ Intensive Care Unit, \\ University Hospital of Patras, Patras, Greece; ${ }^{3}$ York Teaching Hospital NHS Foundation Trust, York, United \\ Kingdom; ${ }^{4}$ Department of Respiratory Medicine, University Hospital of Patras, Patras, Greece; ${ }^{5}$ Intensive \\ Care Unit, Brugmann University Hospital, Brussels, Belgium; ${ }^{6}$ Department of Anesthesiology, Washington \\ University School of Medicine, St. Louis, Missouri, USA
}

\begin{abstract}
The objective of this study is to assess the prognostic value regarding 28-day outcome of the quick sequential organ failure assessment (qSOFA) score and the combined score calculated from blood lactate levels + qSOFA (LqSOFA) score in elderly
\end{abstract}

\begin{abstract}
Correspondence: Menelaos Karanikolas, Department of Anesthesiology, Washington University School of Medicine, Campus Box 8054, 660 S. Euclid Avenue, St. Louis, Missouri 63110, USA. Tel. +1.314.3622340.

E-mail: menelaos.karanikolas@gmail.com

Key words: Sepsis; Lactate; qSOFA score; LqSOFA score; Emergency Department; Mortality.

Contributions: DV treated the patients, collected data, reviewed the literature and wrote the manuscript; VK treated the patients, did statistical analysis and revised the manuscript; NDP, LK, CP collected data and reviewed the literature; $\mathrm{MK}$ reviewed the literature, analyzed data and revised the manuscript; CG supervised the study and revised the manuscript.
\end{abstract}

Conflict of interest: the authors declare no potential conflict of interest.

Funding: this work was supported solely by Department funds from the Department of Internal Medicine, University Hospital of Patras, Greece.

Data availability statement: the anonymized raw data used to support the findings of this study are available as an Excel spreadsheet from the first author upon request.

Received for publication: 1 April 2019.

Revision received: 2 September 2019.

Accepted for publication: 18 September 2019.

This work is licensed under a Creative Commons Attribution 4.0 License (by-nc 4.0).

C Copyright: the Author(s), 2019

Licensee PAGEPress, Italy

Emergency Care Journal 2019; 15:8235

doi:10.4081/ecj.2019.8235 patients initially treated in the Emergency Department (ED) for sepsis due to pneumonia or other chest infections. This is a prospective observational study, conducted at the ED in a Greek University Hospital. Forty-one patients with sepsis due to chest infection were enrolled in the study. All patients were treated in the Resuscitation Room of the ED according to the international treatment protocols for sepsis. The qSOFA score was calculated on admission for all patients, and one point was added in the calculation of the LqSOFA score in patients with blood lactate levels >2 $\mathrm{mmol} / \mathrm{L}$. Both the qSOFA and the LqSOFA scores had high sensitivity and specificity in predicting unfavorable outcome in elderly patients with chest infection and sepsis. In the ongoing debate of early diagnosis of sepsis and identification of prognostic indexes of the syndrome, qSOFA score alone or in combination with lactate levels could serve as a reliable predictor of outcome. Large prospective studies are needed to further evaluate the role and prognostic validity of these scores in the ED.

\section{Introduction}

Sepsis remains one of the leading causes of death worldwide. Based on the latest 2016 definition, sepsis is now diagnosed as a life-threatening organ dysfunction caused by a dysregulated host response to an infection. ${ }^{2}$ The necessity of new definitions for sepsis and septic shock was due to the complex underlying pathophysiology, however, a diagnostic gold standard still does not exist. Among several commonly used biomarkers and prognostic scores, quick sequential organ failure assessment (qSOFA) score is increasingly used in the evaluation of patients with suspected sepsis in medical settings like the Emergency Department (ED). After the introduction of the qSOFA in 2016 by the Task force, its utility as a prognostic tool remains a matter of debate, ${ }^{3,4}$ We present a case series of 41 elderly patients suffering from chest infection who were initially treated in the Resuscitation Room of the Emergency Department in a Greek tertiary care University Medical Center. qSOFA score and a score calculated by the combination of qSOFA and blood lactate levels (LqSOFA score) at admission time were used as predictors for the 28-day outcome. 


\section{Materials and Methods}

\section{Study population and design}

We enrolled in the study 41 consecutive patients who presented in the ED of the University Hospital of Patras, Greece with diagnosis of sepsis due to chest infection and received initial treatment in the Acute Care Area of the ED between January 01, 2018 and June 30, 2018. Inclusion criteria were age $>18$ years and diagnosis of pneumonia confirmed by clinical examination, arterial blood gas analysis and radiographic findings (chest X-ray) performed in the ED. Exclusion criteria were known liver or renal disease, acute renal failure with creatinine clearance $<50$ $\mathrm{mL} / \mathrm{min} / 1.73 \mathrm{~m}^{2}$ ), metformin use and cardiovascular instability requiring use of intravenous vasoactive agents on admission.

The diagnosis of sepsis was based on the international criteria and sepsis-3 definitions. ${ }^{2}$ All patients were initially treated for respiratory distress, as defined by increased respiratory rate $(\mathrm{RR}>30)$, and abnormal values in partial oxygen pressure $\left(\mathrm{PaO}_{2}\right)$ and/or carbon dioxide partial oxygen pressure $\left(\mathrm{PaCO}_{2}\right)$ in arterial blood gas analysis. Patients received oxygen therapy via a Venturi mask or received support with forced oxygenation using Continuous Positive Airway Pressure. All appropriate treatment was provided in accordance with the Surviving Sepsis Guidelines. ${ }^{5}$ After stabilization, all patients were admitted to the Internal Medicine Department of the Hospital for further evaluation and treatment.

The study protocol was approved by the Ethics Committee of the University Hospital and all patients (or their legal guardian or power of attorney) gave written informed consent for participation in the study. All data collected for the purposes of this study were anonymized and stored in an encrypted electronic database. The medical team involved in the study included University of Patras Faculty physicians, resident physicians rotating through the ED and outside consultants.

\section{Data collection}

Collected data included demographic information, a detailed history of present illness, pre-existing co-morbidities and related medications, social history and surgical history. Clinical records for each patient consisted of detailed physical examination and radiological data (chest X-ray images). Blood laboratory data were obtained immediately on admission to the ED after analyzing 10 $\mathrm{mL}$ of peripherally collected blood samples, blood gas analysis also was obtained in order to measure lactate levels and monitor respiratory status. For each patient, the qSOFA score was calculated based on the following variables: i) systolic blood pressure $\leq 100 \mathrm{mmHg}$; ii) $\mathrm{RR} \geq 22$ breaths/min; and iii) altered mental status. One point was added for every abnormal value from above parameters in order to calculate the score. ${ }^{6}$

Serial serum lactate measurements were obtained in the ED at the time of initial assessment and were repeated at the discretion of the physician team in order to monitor response to treatment. The first serum lactate value was used to calculate the LqSOFA score by adding 1 point to the qSOFA score for lactate levels $>2 \mathrm{mmol} / \mathrm{L}$. Patient demographic data, major laboratory findings, qSOFA score and LqSOFA scores (based on the aggregation of qSOFA score + lactate blood levels) are presented in Table 1. Patient survival to hospital discharge was the primary endpoint of the study.

\section{Statistical analysis}

Data were analyzed with descriptive and inferential statistics. Continuous variables were summarized as mean $( \pm \mathrm{SD})$ and differences between groups were evaluated using the Student's t-test or Mann-Whitney U test as appropriate. Frequency of comorbidities in survivors vs non-survivors was compared using the Chi-square test. Variables which differed significantly between survivors and non-survivors in univariate analysis (age, dementia, qSOFA and LqSOFA) were then evaluated as independent predictors of outcome using logistic regression analysis. However, because of collinearity, dementia could not be entered in the regression model and only one of the qSOFA or LqSOFA variables (but not both) could be entered in each regression analysis.

The discriminating ability of qSOFA and LqSOFA for predicting mortality was expressed as the area under the receiver operating characteristic curve (ROC).

All tests were two-tailed, and $\mathrm{P}<0.05$ were considered statistically significant. All data were analyzed using the IBM SPSS Statistics software (version 25) and the MedCalc Statistical Software version 19.0.7 (MedCalc Software bvba, Ostend, Belgium).

\section{Results}

The study enrolled 41 patients ( 24 women, 17 men) with age

Table 1. Demographic, basic laboratory data, clinical data and calculated severity scores in survivors vs non-survivors. Values are presented as Mean (SD) or as number of patients.

\begin{tabular}{|c|c|c|c|}
\hline & Survivors (N=19) & Non-survivors ( $\mathrm{N}=22)$ & P-value \\
\hline Gender (M/F) & $9 / 10$ & $8 / 14$ & ns \\
\hline Age & $74(12)$ & $83.5(7.3)$ & 0.027 \\
\hline WBC & $17400(9000)$ & $15500(7000)$ & ns \\
\hline CRP & $12.5(10)$ & $15.2(10)$ & ns \\
\hline Central Line in ED & 8 & 17 & 0.021 \\
\hline Vasopressors in ED & 0 & 4 & ns \\
\hline Dementia & 3 & 13 & 0.001 \\
\hline Stroke & 1 & 3 & ns \\
\hline COPD & 3 & 2 & ns \\
\hline Serum lactate level & $2.7(1.1)$ & $3(1.16)$ & ns \\
\hline qSOFA & $1.06(0.64)$ & $1.9(0.5)$ & $<0.001$ \\
\hline LqSOFA & $1.72(0.83)$ & $3(0.66)$ & $<0.001$ \\
\hline
\end{tabular}

WBC, white blood cells; CRP, C-reactive protein; ED, Emergency Department; COPD, chronic obstructive pulmonary disease; qSOFA, quick SOFA; LqSOFA, lactate qSOFA; ns, not significant. 
range 55 to 98 years. The primary diagnosis on arrival to the ED was pneumonia confirmed by clinical signs (increased respiratory ratio, dyspnea, fever, respiratory distress) and radiologic findings on chest X-ray in all patients. In addition, detailed evaluation also revealed the presence of Cholecystitis in 7 patients, Diverticulitis in 1 patient, Cellulitis in 3 patients, Glomerulonephritis in 2 patients, Osteomyelitis in 1 patient, Pyelonephritis in 1 patient and Urinary Tract Infection in 3 patients. Demographic data, laboratory data and calculated qSOFA and LqSOFA scores are summarized in Table 1. Non-survivors were significantly older, required central line significantly more often, had significantly higher frequency of dementia and had significantly higher qSOFA and LqSOFA scores compared to survivors.

Furthermore, in order to better understand the impact of qSOFA score and of Lactate values on mortality, we divided patients in four subgroups based on whether they had qSOFA score $<2 v s \geq 2$, and Lactate values $\leq 2 v s>2$, and calculated mortality for these subgroups. The data are summarized in Table 2. Chi-square analysis of these subgroups showed that differences in mortality were highly significant $(\mathrm{P}<0.00001)$.

ROC analysis was used to distinguish patients who survived from patients who did not survive and showed that the qSOFA score area under the curve (AUC) was 0.849 (95\% CI 0.703-0.942; $\mathrm{P}<0.0001$; Figure 1 ). qSOFA $\geq 2$ had $90.9 \%$ sensitivity and $78.9 \%$ specificity in predicting unfavorable outcome. Similarly, the LqSOFA score $>2$ AUC was 0.830 (95\% CI 0.679-0.929; $\mathrm{P}<0.0001$; Figure 2), and LqSOFA $>2$ had $81.8 \%$ sensitivity and $84.2 \%$ specificity in predicting unfavorable outcome.

Multivariate logistic regression analysis showed that age was not a significant predictor of mortality, whereas qSOFA $\geq 2$ and LqSOFA $>2$ were both significant predictors: patients with qSOFA score $\geq 2$ or LqSOFA score $>2$ had significantly higher risk of mortality (OR 32.15, 95\% CI 4.971 to $207.919, \mathrm{P}<0.001$ and OR $25.213,95 \%$ CI 4.239 to $149.978, \mathrm{P}<0.001$ respectively Tables 3 and 4).

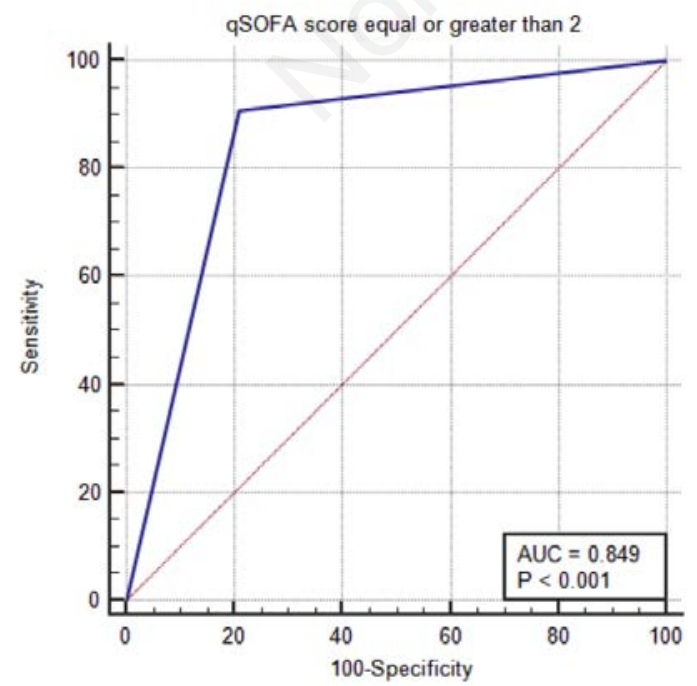

Figure 1. ROC curve evaluating the ability of $\mathrm{qSOFA} \geq 2$ to predict mortality. Area under the curve $(A U C)=0.849, P<0.001$.

\section{Discussion and Conclusions}

In this study, we assessed the predictive value of the qSOFA and the calculated LqSOFA score in a prospective case series of sepsis patients with chest infection. Although several scores (SOFA, APACHE II, SAPS II) and serum biomarkers (white blood cell count, C-reactive protein, Procalcitonin) have been used by clinicians for assessment of patients with suspected or proven sepsis, a gold standard for diagnosis and monitoring of the sepsis syndrome has not been established, and the sensitivity and specificity of these scores and biomarkers for prediction of outcome vary. ${ }^{7,8}$ The qSOFA score is easy to calculate using three bedside parameters and has been recently proposed as a rapid test for detection of sepsis in early stages. Although the majority of publications show that qSOFA score has better accuracy than SIRS criteria for predicting mortality in the non-intensive care unit (ICU) environment, ${ }^{9,10}$ there is ongoing debate regarding its usefulness because of poor sensitivity. ${ }^{11}$ A meta-analysis published by Jiang et al. in 2018 attempted to compare the qSOFA score with the SIRS criteria and determine the accuracy of the qSOFA score in predicting mortality in ED patients with infections and showed that although qSOFA score $\geq 2$ and SIRS score $\geq 2$ were both strongly

Table 2. Mortality in patient sub-groups, based on combination of qSOFA and Lactate values. The observed differences in mortality between the four sub-groups are highly significant $(\mathbf{P}<0.00001)$.

\begin{tabular}{lcccc} 
Sulb-Group Definition & Number of patients & Deaths & Mortality \\
qSOFA $\leq 1$ & Lactate $\leq 2$ & 6 & 0 & $0 \%$ \\
qSOFA $=1$ & Lactate $>2$ & 11 & 2 & $18.2 \%$ \\
\hline qSOFA $\geq 2$ & Lactate $\leq 2$ & 3 & 2 & $66.6 \%$ \\
qSOFA $\geq 2$ & Lactate $>2$ & 21 & 18 & $85.7 \%$ \\
\hline Total & & 41 & 22 & - \\
\hline
\end{tabular}

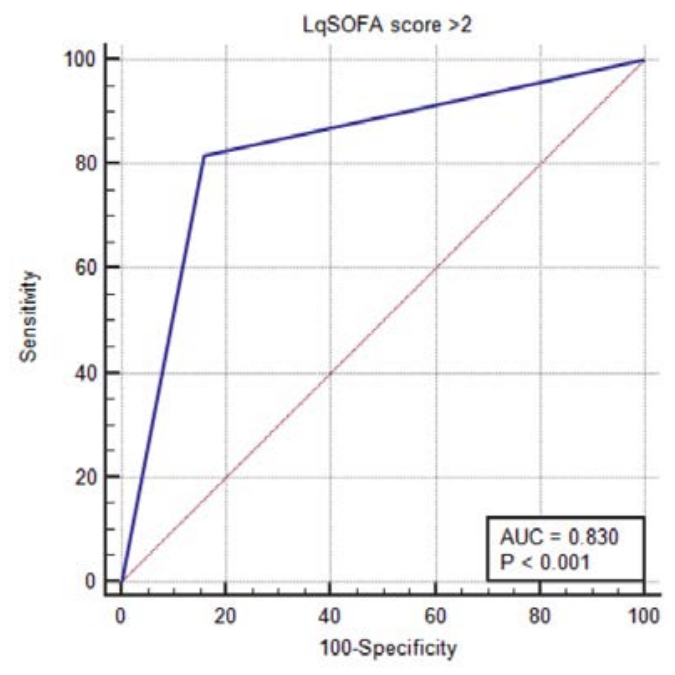

Figure 2. ROC curve evaluating the ability of LqSOFA $>2$ to predict mortality. Area under the curve $(A U C)=0.830, P<0.001$. 
Table 3. Logistic regression analysis evaluating $\mathrm{qSOFA} \geq 2$ as predictor of risk of death in septic patients. qSOFA $\geq 2$ is significant predictor of mortality both in univariate and in multivariate analysis.

\begin{tabular}{lcccccc} 
& & Univariate Analysis & Pr & Multivariate Analysis & P-value \\
Age & OR & $95 \%$ CI & P-value & OR & $95 \%$ Cl & 0.208 \\
qSOFA 22 & 1.082 & 1.008 to 1.161 & 0.03 & 1.059 & 0.969 to 1.158 & $<.001$ \\
\hline
\end{tabular}

$\mathrm{OR}$, odds ratio; $\mathrm{Cl}$, confidence interval.

Table 4. Logistic regression analysis evaluating LqSOFA $>2$ as predictor of risk of death in septic patients. LqSOFA $>2$ is significant predictor of mortality both in univariate and in multivariate analysis.

\begin{tabular}{lcccccc} 
& & Univariate Analysis & & \multicolumn{2}{c}{ Multivariate Analysis } \\
& OR & $95 \%$ CI & P-value & OR & 95\% CI & 0.992 to 1.186 \\
Age & 1.082 & 1.008 to 1.161 & 0.03 & 1.084 & 0.075 \\
LqSOFA $>2$ & 24 & 4.649 to 123.904 & $<0.001$ & 25.213 & 4.239 to 149.978 & $<0.001$ \\
\hline
\end{tabular}

$\mathrm{OR}$, odds ratio; $\mathrm{Cl}$, confidence interval.

associated with mortality, the qSOFA cannot completely replace the use of SIRS in the ED due to its low sensitivity. ${ }^{12}$

Septic shock is defined, according to the new definitions as a subset of sepsis in which the patient has profound hypoperfusion. Septic shock is characterized by underlying circulatory and metabolic abnormalities resulting in tissue hypoperfusion with serum lactate level $\geq 2 \mathrm{mmol} / \mathrm{L}$ despite adequate volume resuscitation and hypotension that requires vasopressors in order to maintain mean arterial pressure $\geq 65 \mathrm{mmHg}$. Increased serum lactate levels can be identified in early stages of the sepsis syndrome due to abnormal tissue perfusion. During the process of the sepsis syndrome, insufficient circulation fails to maintain adequate tissue metabolism. Elevated blood lactate levels most commonly indicate impaired oxidative phosphorylation secondary to decreased oxygen availability to the tissue cells (hypoxic hypoxia) and/or tissue hypoperfusion. This complex pathophysiology leads finally to tissue hypoxia, and lactate overproduction due to increased anaerobic glycolysis. ${ }^{13,14}$ However, there is evidence supporting the view that, in addition to tissue hypoxia and anaerobic glycolysis, other mechanisms are also involved in sepsis-related hyperlactatemia. Experimental and human studies all consistently support that elevated lactate levels in sepsis are explained by increased aerobic glycolysis secondary to activation of the stress response (adrenergic stimulation) in order to facilitate a bioenergetic efficiency. ${ }^{15}$

Literature search revealed a few earlier publications related to lactate measurements and qSOFA score evaluation in the Emergency Department: A multi-center retrospective cohort study from US hospitals enrolled 3743 patients assessed in the ED and revealed that qSOFA criteria performed as well or better compared to SIRS criteria, severe sepsis criteria and lactate levels in predicting critical illness. ${ }^{16}$ A retrospective cohort study from Shetty et al. included 12555 patients with suspected infection in Australia and the Netherlands and showed that the lactate $\geq 2 \mathrm{mmol} / \mathrm{L}$ thresholdbased LqSOFA score performs better than qSOFA alone in identifying the risk of adverse outcomes (defined as mortality and/or ICU stay $\geq 72 \mathrm{~h}$ ) in ED patients. ${ }^{6}$ A retrospective study published in 2018 by Jung included 457 surgical patients with complicated intra-abdominal infection and concluded that the LqSOFA score, which is derived from the qSOFA and serum lactate levels had better predictive performance and higher sensitivity regarding mortality than the qSOFA alone and had predictive performance comparable the full SOFA score. ${ }^{17}$
Our results suggest that both the qSOFA and the LqSOFA scores calculated on admission to the Emergency Department had high sensitivity and specificity in predicting mortality in patients with chest infection and sepsis. However, comparison of the two scores as predictors of outcome shows that qSOFA has higher sensitivity (90.9\% vs $81.8 \%$ ), whereas LqSOFA has higher specificity $(84.2 \%$ vs $78.9 \%)$. Because there is a need for effective reliable instruments to detect sepsis in the Emergency Department, where treatment in the golden hour ${ }^{18}$ remains the cornerstone of therapy, use of additional modified indexes as means to improve sensitivity or specificity of detecting patients at risk for adverse outcome could be helpful.

Limitations of our study include the small sample size and the fact that it only includes data from elderly patients treated for respiratory failure in the Resuscitation room of the Emergency Department. The dynamic nature of the sepsis syndrome can make detection of sepsis difficult in the ED setting, and the qSOFA score alone has limitations in predicting outcome due to low sensitivity. The combination of qSOFA score with other clinical parameters and laboratory measurements could possibly identify more patients with sepsis who may benefit from further assessment and interventions in the ED. This clinical study suggests that serum lactate level, a sensitive finding reflecting anaerobic metabolism has comparable validity when added to qSOFA score, and its use in the calculation of prognostic scores already in use could be beneficial in clinical practice by improving specificity of predicting a bad outcome. However, because of the significant limitations of our study, further large prospective clinical studies are warranted to confirm and validate our findings.

\section{References}

1. Martin GS, Mannino DM, Eaton S, et al. The epidemiology of sepsis in the United States from 1979 through 2000. N Engl J Med 2003;348:1546-54.

2. Singer M, Deutschman CS, Seymour CW, et al. The third international consensus definitions for sepsis and septic shock (sepsis-3). JAMA 2016;315:801-10.

3. Dorsett M, Kroll M, Smith CS, et al. Qsofa has poor sensitivity for prehospital identification of severe sepsis and septic shock. 
Prehosp Emerg Care 2017;21:489-97.

4. Williams JM, Greenslade JH, McKenzie JV, et al. Systemic inflammatory response syndrome, quick sequential organ function assessment, and organ dysfunction: Insights from a prospective database of ed patients with infection. Chest 2017;151:586-96.

5. Rhodes A, Evans LE, Alhazzani W, et al. Surviving sepsis campaign: International guidelines for management of sepsis and septic shock: 2016. Intensive Care Med 2017;43:304-77.

6. Shetty A, MacDonald SP, Williams JM, et al. Lactate $>/=2$ $\mathrm{mmol} / \mathrm{l}$ plus qsofa improves utility over qsofa alone in emergency department patients presenting with suspected sepsis. Emerg Med Australas 2017;29:626-34.

7. Seymour CW, Liu VX, Iwashyna TJ, et al. Assessment of clinical criteria for sepsis: For the third international consensus definitions for sepsis and septic shock (sepsis-3). JAMA 2016;315:762-74.

8. Wang Y, Wang D, Fu J, et al. [Predictive value of sofa, qsofa score and traditional evaluation index on sepsis prognosis]. Zhonghua Wei Zhong Bing Ji Jiu Yi Xue 2017;29:700-4.

9. Finkelsztein EJ, Jones DS, Ma KC, et al. Comparison of qsofa and sirs for predicting adverse outcomes of patients with suspicion of sepsis outside the intensive care unit. Crit Care 2017;21:73.

10. Park HK, Kim WY, Kim MC, et al. Quick sequential organ failure assessment compared to systemic inflammatory response syndrome for predicting sepsis in emergency department. J Crit Care 2017;42:12-7.
11. Askim A, Moser F, Gustad LT, et al. Poor performance of quick-sofa (qsofa) score in predicting severe sepsis and mortality - a prospective study of patients admitted with infection to the emergency department. Scand J Trauma Resusc Emerg Med 2017;25:56.

12. Jiang J, Yang J, Mei J, et al. Head-to-head comparison of qsofa and sirs criteria in predicting the mortality of infected patients in the emergency department: A meta-analysis. Scand J Trauma Resusc Emerg Med 2018;26:56.

13. Kellum JA, Kramer DJ, Lee K, et al. Release of lactate by the lung in acute lung injury. Chest 1997;111:1301-5.

14. Shapiro NI, Howell MD, Talmor D, et al. Serum lactate as a predictor of mortality in emergency department patients with infection. Ann Emerg Med 2005;45:524-8.

15. Garcia-Alvarez M, Marik P, Bellomo R. Sepsis-associated hyperlactatemia. Crit Care 2014;18:503.

16. Rodriguez RM, Greenwood JC, Nuckton TJ, et al. Comparison of qsofa with current emergency department tools for screening of patients with sepsis for critical illness. Emerg Med J 2018;35:350-6.

17. Jung YT, Jeon J, Park JY, et al. Addition of lactic acid levels improves the accuracy of quick sequential organ failure assessment in predicting mortality in surgical patients with complicated intra-abdominal infections: A retrospective study. World J Emerg Surg 2018;13:14.

18. Rivers E, Nguyen B, Havstad S, et al. Early goal-directed therapy in the treatment of severe sepsis and septic shock. N Engl J Med 2001;345:1368-77. 